\title{
Ambulant monitoring of bladder oxygenation and hemodynamics using wireless near-infrared spectroscopy
}

\author{
Andrew John Macnab, MD (London), FRCPC, FRCPCH, FCAHS, ${ }^{*}$ Babak Shadgan, MD, MSc, PhD; ${ }^{\dagger}$ \\ Lynn Stothers, MD, MHSc, FRCSC; ${ }_{;}^{*}$ Kourosh Afshar, MD, FRCSC ${ }^{\S}$
}

*Department of Urology, Faculty of Medicine, University of British Columbia, UBC Hospital Bladder Care Centre, Vancouver BC; +UBC Bladder Care Centre, University of British Columbia, Vancouver BC; $\S$ Department of Urology, British Columbia's Children's Hospital, Vancouver, BC

Cite as: Can Urol Assoc J 2013;7:E98-E104. http://dx.doi.org/10.5489/cuai.271. Epub 2012 January 24.

\section{Abstract}

Introduction: Near-infrared spectroscopy (NIRS) non-invasively detects changes in the concentration of the chromophores oxygenated $\left(\Delta \mathrm{O}_{2} \mathrm{Hb}\right)$ and deoxygenated hemoglobin $(\Delta \mathrm{HHb})$ as the bladder detrusor muscle contracts during voiding. Such data provide novel information on bladder oxygenation and hemodynamics. We evaluated the feasibility of monitoring ambulant subjects using a wireless NIRS device.

Methods: The wireless device uses paired light-emitting diodes (wavelengths 760 and $850 \mathrm{~nm}$ ) and a silicon photodiode detector. We monitored 14 asymptomatic subjects ( 10 adults, 4 children) and 6 symptomatic children with non-neurogenic lower urinary tract dysfunction (NLUTD) during spontaneous voiding after natural filling. The device was taped to the abdominal skin $2 \mathrm{~cm}$ above the symphysis pubis across the midline. The wireless NIRS data (patterns of change in chromophore concentration) were compared between subjects and to the data obtained using a laser-powered instrument.

Results: Graphs of $\Delta \mathrm{O}_{2} \mathrm{Hb}, \Delta \mathrm{HHb}$ and total hemoglobin $(\Delta \mathrm{tHb})$ were obtained from all 20 patients. Data during uroflow showed reproducible patterns of bladder chromophore change between asymptomatic subjects (rise in $\Delta \mathrm{tHb} / \Delta \mathrm{O}_{2} \mathrm{Hb}$ ), consistent with laser instrument data. In contrast, all 6 symptomatic children had a negative trend in $\Delta \mathrm{tHb}$, with falls in $\Delta \mathrm{O}_{2} \mathrm{Hb}$. One adult experienced "shy" bladder and changes in hemodynamics/oxygenation occurred while bladder volume was unchanged.

Conclusions: Wireless NIRS bladder monitoring is feasible in ambulant adults and children; wireless and laser-derived data in asymptomatic subjects are comparable. Pilot data suggest that subjects with symptomatic NLUTD have impaired bladder oxygenation/ hemodynamics. The fact that chromophore changes occur when bladder volume remains constant supports the concept that NIRS data are a physiologic measure.

\section{Introduction}

Changes in tissue oxygenation and hemodynamics can be monitored using near-infrared spectroscopy (NIRS) by following variations in concentration of the chromophores oxygenated hemoglobin $\left(\Delta \mathrm{O}_{2} \mathrm{Hb}\right)$ and reduced hemoglobin $(\Delta \mathrm{HHb})$ and changes in total hemoglobin $(\Delta \mathrm{tHb})\left[\mathrm{O}_{2} \mathrm{Hb}+\right.$ $\mathrm{HHb}$. There are many reports on the physics principles involved, the differences in absorption spectra of hemoglobin when oxygenated and deoxygenated, the range of research studies where NIRS technology has been successfully applied..$^{1-3}$ In brain and muscle clinical applications, the patterns of chromophore changes differ in healthy and diseased humans according to the physiologic consequences of disease pathology. ${ }^{1,2}$ Recent urologic applications of NIRS include research using single-channel continuous wave $^{4}(\mathrm{CW})$ and functional NIRS ${ }^{5}$ (fNIRS) methodologies to monitor changes in chromophore concentration within the bladder detrusor as the organ fills and empties. NIRS monitoring can be done simultaneously with pressure flow studies (urodynamic studies [UDS]), and provides urologists with previously unavailable insights into the hemodynamics of the detrusor microcirculation and oxygen supply and demand in the context of voiding. ${ }^{6}$

In men with lower urinary tract symptoms (LUTS) simultaneous UDS and NIRS studies identified different patterns of change in $\Delta \mathrm{O}_{2} \mathrm{Hb}$ and $\Delta \mathrm{tHb}$ in those with and without bladder outlet obstruction (BOO). In addition to the physiologic information provided by the NIRS data, two diagnostic algorithms incorporating NIRS parameters gave comparable discriminant ability for the presence or absence of $\mathrm{BOO}$ as the Abrahams-Griffiths nomogram. ${ }^{4,7}$ Independent investigators $^{8}$ initially validated the diagnostic ability of the original algorithm which incorporates the trend of $\Delta \mathrm{O}_{2} \mathrm{Hb}$ (positive or negative) $\mathrm{Q}_{\max }$ and residual volume. ${ }^{4}$ Recently, Chung and colleagues' second study suggested the algorithm's $\Delta \mathrm{O}_{2} \mathrm{Hb}$ trend component warrants improvement. ${ }^{9}$ The sec- 
ond algorithm, which uses NIRS data alone, employs classification and regression tree analysis (CART) and has better discriminant ability than the first ${ }^{7}$ the CART application is described to help interested clinicians. ${ }^{10}$ The diagnostic ability of NIRS in patients with detrusor overactivity (DO) has also been reported, ${ }^{11}$ and the potential for NIRS to improve pelvic floor training has been hypothesized. ${ }^{12}$

Currently most NIRS instruments require lasers and fibre optic cables. ${ }^{3,4,13}$ But light-emitting diodes (LEDs) are an alternative light source, and make a small, wireless, selfcontained instrument feasible. This would be particularly beneficial in children, where non-invasive methods for evaluation of bladder muscle function are being sought, and in patients with neurogenic bladder to make ambulant or long-term urologic monitoring possible.

We report the feasibility of using a miniature wireless NIRS device to monitor the bladder in ambulant subjects.

\section{Methods}

\section{Instrumentation}

The wireless device (Portamon, Artinis Medical Technologies, BV, The Netherlands) was designed for muscle exercise physiology protocols. ${ }^{14}$ Its key technical features include: miniaturization (dimensions $83 \times 52 \times 20 \mathrm{~mm}$; weight $84 \mathrm{~g}$ ); 3 paired LEDs as the NIR light source (LED wavelengths 760 and $850 \mathrm{~nm}$ ); a silicon photodiode detector screened against ambient light; data collection at $10 \mathrm{~Hz}$; a rechargeable lithium polymer battery; a 2-mb internal memory; and Bluetooth technology.

Raw optical data can be stored during ambulant measurement or broadcast to a laptop computer; graphs to illustrate changes in $\Delta \mathrm{O}_{2} \mathrm{Hb}, \Delta \mathrm{HHb}$ and $\Delta \mathrm{tHb}\left[\mathrm{O}_{2} \mathrm{Hb}+\mathrm{HHb}\right]$ can be plotted.

\section{Subjects}

We recruited a convenience sample of 10 healthy asymptomatic adult males (age range: $24-65$ years) and 10 children (4 asymptomatic and 6 symptomatic attending a University clinic for investigation of non-neurogenic lower urinary tract dysfunction non-neurogenic lower urinary tract dysfunction (NLUTD) [age range: 5-11 years]). Urine microscopy and cultures were not part of the evaluation of symptomatic subjects at this visit (all had had negative tests previously); also no patients were on antimuscarinics. We obtained signed consent forms and our study was approved by our University ethical review board.

\section{Protocol}

Each subject spontaneously emptied his/her bladder, and then had water to drink ad libitum so that the bladder could fill naturally. The device was attached to the abdominal wall using medical adhesive tape with the emitters/sensor $2 \mathrm{~cm}$ superior to the symphysis pubis and across the midline, and secured by a retaining strap. Subjects were then able to be fully ambulant and in a separate room remote from the investigator. Asymptomatic subjects were studied with NIRS during uroflow. In symptomatic children NIRS was conducted simultaneously with the regular urologic studies planned for the clinic visit (uroflow and EMG).

Changes in chromophore concentration in the bladder detrusor during spontaneous bladder emptying were monitored with pre- and post-voiding baseline data collection. To void, adult subjects were asked to stand still and children to sit; permission was then given to empty the bladder, with the instruction to do so normally without abdominal straining.

Graphs of change in $\Delta \mathrm{O}_{2} \mathrm{Hb}, \Delta \mathrm{HHb}$ and $\Delta \mathrm{tHb}$ from baseline timed at permission to void were compared between subjects; also, trends of change (positive or negative) were compared between asymptomatic and symptomatic children. The asymptomatic adult data was also compared to data collected using a conventional laser-powered instrument and with the same study protocol and software algorithm.

\section{Results}

Complete NIRS data sets were obtained for all 20 subjects. No technical difficulties were encountered, and all subjects were able to be ambulant, except during the voiding segment of the protocol. During uroflow, the patterns of change in chromophore concentration were comparable in all 14 asymptomatic subjects. One adult had a "shy bladder" and experienced a delay following permission to void that lasted 137 seconds; however, from uroflow start, his pattern of chromophore change matched other asymptomatic subjects. The 9 adults and 4 children who voided promptly showed an initial increase in $\Delta \mathrm{tHb}$ after permission to void and a progressive rise during uroflow up to the point of peak urine flow, with a gradual decline thereafter. The increase in $\Delta \mathrm{tHb}$ was predominantly due to an increase in $\Delta \mathrm{O}_{2} \mathrm{Hb}$. This pattern of change was comparable to data collected in normal adults using a laser powered NIRS instrument in prior studies. ${ }^{13}$ Representative graphs of this pattern from the wireless/LED device and a conventional/laser instrument are available (Fig. 1).

In the adult with the "shy" bladder, the typical brief initial rise in $\Delta \mathrm{tHb}$ and $\Delta \mathrm{O}_{2} \mathrm{Hb}$ following permission to void was evident, however during the subsequent delay prior to uroflow variations in detrusor hemodynamics and oxygen, supply and demand were evident, while his bladder 


\section{A. Wireless LED Device}

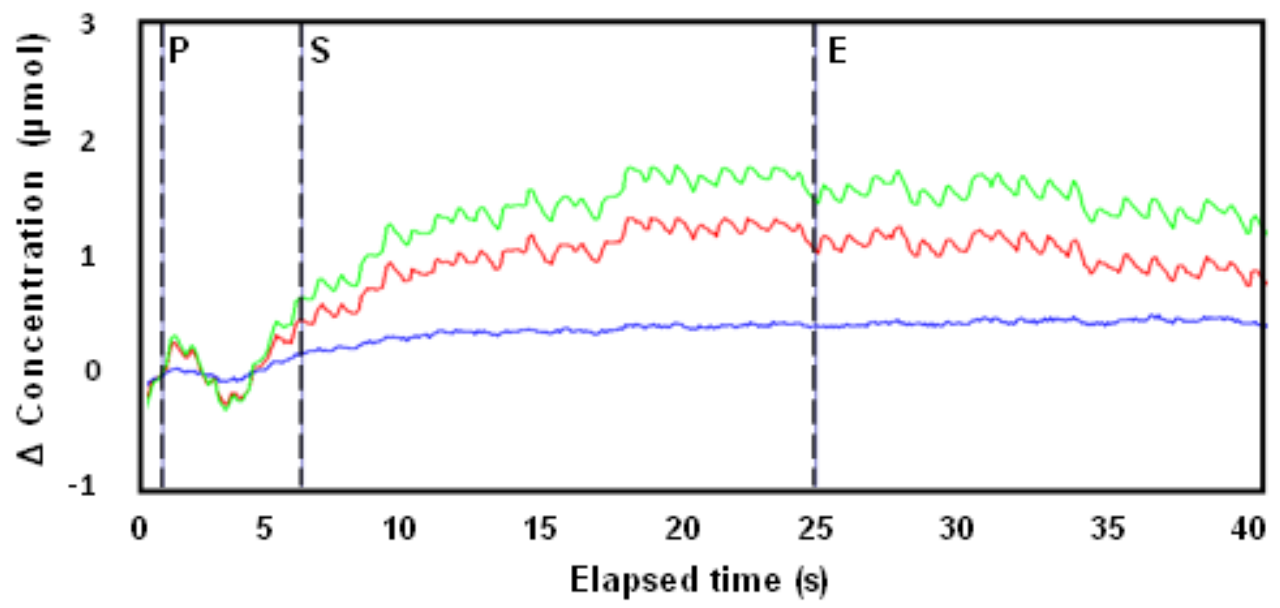

\section{B- Conventional Laser Device}

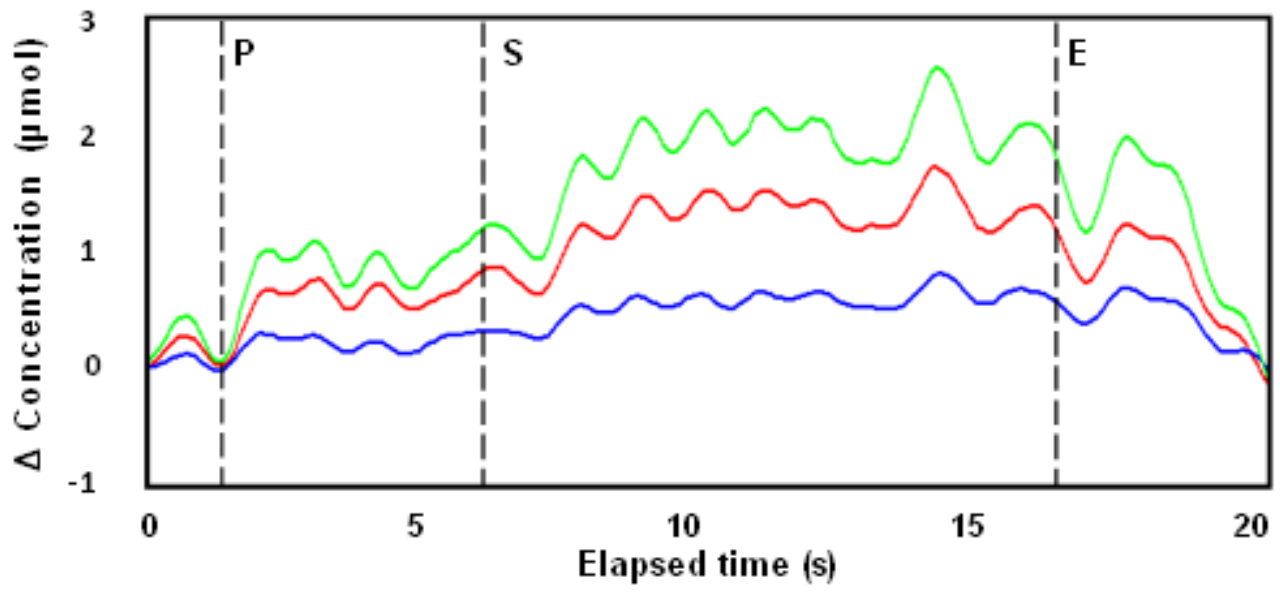

P-Permission to void, S- Urine flow starts, E- Uroflow ends

Total Hb

Fig. 1. Changes in chromophore concentration from permission to void to uroflow end in asymptomatic adults obtained with A: The wireless NIRS device with light emitting diodes as the NIR source; and B: A conventional laser powered NIRS instrument. Both graphs show the pattern of chromophore change common to asymptomatic subjects: an increase in $\Delta \mathrm{tHb}$ following permission to void predominantly reflecting an increase in $\Delta \mathrm{O}_{2} \mathrm{Hb}$, then during uroflow $\Delta \mathrm{tHb}$ and $\Delta \mathrm{O}_{2} \mathrm{Hb}$ continue to increase, while the deoxygenated hemoglobin concentration shows minimal change or no change.

volume remained constant. We have illustrated data during this period with two episodes of movement-generated artifact removed to clarify the data trends (Fig. 2). From 32 seconds, $\Delta \mathrm{tHb}$ and $\Delta \mathrm{O}_{2} \mathrm{Hb}$ progressively rose as urge without uroflow continued. This indicated an increase in oxygenated blood volume in the detrusor microcirculation. From 52 seconds, $\Delta \mathrm{O}_{2} \mathrm{Hb}$ declined and $\Delta \mathrm{HHb}$ progressively rose. This indicated an increased rate of oxygen consumption, with the fall in $\Delta \mathrm{tHb}$ from 77 to 92 seconds indicating an associated fall in blood volume. From 92 seconds, $\mathrm{tHb}$ rose, predominantly due to increasing $\mathrm{HHB}$, and $\Delta \mathrm{HHb}$ became greater than $\Delta \mathrm{O}_{2} \mathrm{Hb}$, indicating oxygen consumption exceeded oxygen supply as ongoing urge without uroflow continued. The $\Delta \mathrm{HHb}$ continued to rise until 135 seconds. However, from 114 seconds, a sustained rise in $\Delta \mathrm{tHb}$ occurred due to a marked increase in $\Delta \mathrm{O}_{2} \mathrm{Hb}$, and $\Delta \mathrm{O}_{2} \mathrm{Hb}$ became greater than $\Delta \mathrm{HHb}$ just prior to uroflow start. This implied a hemodynamic response with provision of increased oxygenated blood volume which was followed by successful voiding. 


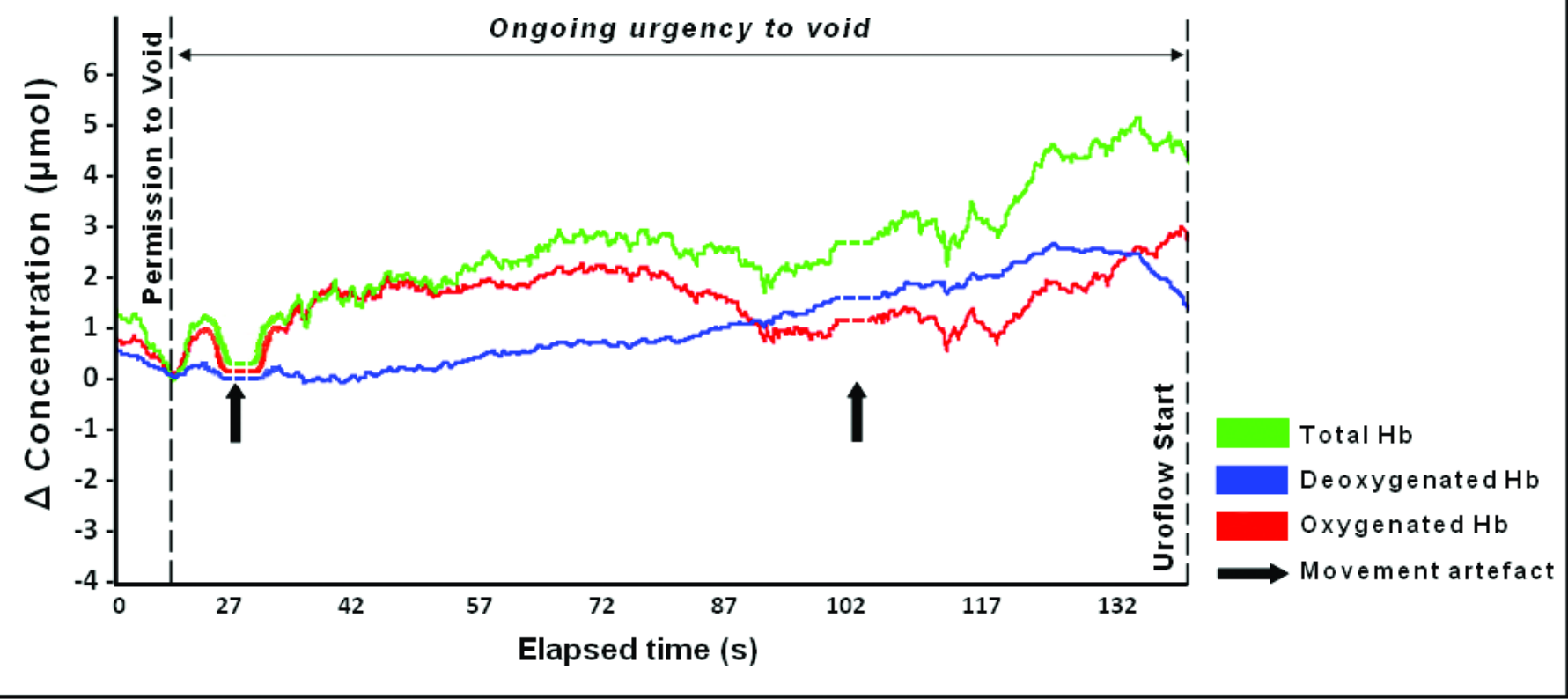

Fig. 2. Changes in chromophore concentration in an asymptomatic adult experiencing voiding delay following permission to void. Spontaneous movements by the subject at 19 and 98 seconds [bold arrows] displaced the data baseline; these data points have been removed [broken line] for clarity of chromophore trends. Changes in blood volume (Total $\mathrm{Hb}$ ) are evident indicating hemodynamic change; the trend of increasing $\Delta \mathrm{HHb}$ indicates rising oxygen consumption; uroflow starts after an abrupt fall in $\Delta \mathrm{HHb}$ that follows a sustained increase in $\Delta \mathrm{tHb} / \Delta \mathrm{O}_{2} \mathrm{Hb}$. Isovolumetric bladder contractions probably occurred during ongoing urgency to void, while bladder volume remained constant.

The 6 children diagnosed with NLUTD ${ }^{15}$ had pilot NIRS data different from the asymptomatic children (Table 1). Fig. 3 shows representative graphs of the pattern of chromophore change from an asymptomatic child (part A) and subjects 1 to 5 (Table 1) with symptoms of NLUTD (part B). In part A, the pattern and positive trend for $\Delta \mathrm{tHb}$ and $\Delta \mathrm{O}_{2} \mathrm{Hb}$ matched those seen in asymptomatic adults (Fig. 1). In part B, there was little or no increase in $\mathrm{tHb}$ following permission to void, and a downward trend was associated with a progressive fall in $\Delta \mathrm{O}_{2} \mathrm{Hb}$ during uroflow. This negative trend implied an overall reduction in the detrusor blood volume and the availability of oxygenated hemoglobin over the course of voiding. Figure 4 shows simultaneous urologic and NIRS data in a symptomatic child (subject 6). Here, abnormalities in uroflow were associated with a simultaneous increase in $\Delta \mathrm{O}_{2} \mathrm{Hb}$ and $\Delta \mathrm{HHb}$ following permission to void and fluctuations in the negative trend in $\Delta \mathrm{tHb}$ during uroflow were due to variations in $\Delta \mathrm{O}_{2} \mathrm{Hb}$.

Insufficient subjects were recruited in this feasibility study to calculate the specificity and sensitivity of the NIRS data, as has been possible in prior studies of patients with and without $\mathrm{BOO}, 4,7,8$ and by Farag and colleagues when using NIRS as a diagnostic entity for DO. ${ }^{11}$

Children readily accepted the device, wore it without complaint and continued to be fully ambulant prior to and after the voiding component of the study. Secure skin attachment was achieved in all adults and children, which prevented movement of the device even when subjects were ambulant.

\begin{tabular}{|c|c|c|c|c|c|c|c|c|}
\hline & Age, yrs & Gender & Weight, kg & BMI & Symptoms & PVR & $\begin{array}{l}\text { Voided } \\
\text { volume, } \mathrm{mL}\end{array}$ & $\begin{array}{c}\text { Average flow, } \\
\mathrm{mL} / \mathrm{sec}\end{array}$ \\
\hline 1 & 3 & Female & 14 & 16.3 & $\begin{array}{c}\text { Severe dysfunction, recurrent UTI, } \\
\text { enuresis }\end{array}$ & 20 & 140 & 5.3 \\
\hline 2 & 10 & Female & 35 & 17.2 & Frequency, urgency & 0 & 91 & 10.1 \\
\hline 3 & 9 & Female & 31 & 17.4 & Recurrent UTI, frequency & 122 & 134 & 8.9 \\
\hline 4 & 14 & Male & 42 & 18.7 & Infrequent voiding, dysuria & 150 & 466 & 7.2 \\
\hline 5 & 7 & Male & 28 & 15.9 & Infrequent voiding, incontinence & 175 & 298 & 5.7 \\
\hline 6 & 5 & Female & 24 & 16.6 & Frequency, incontinence & 19 & 190 & 8.2 \\
\hline
\end{tabular}

BMI: body mass index; PVR: post-void residual; UTI: urinary tract infection. 


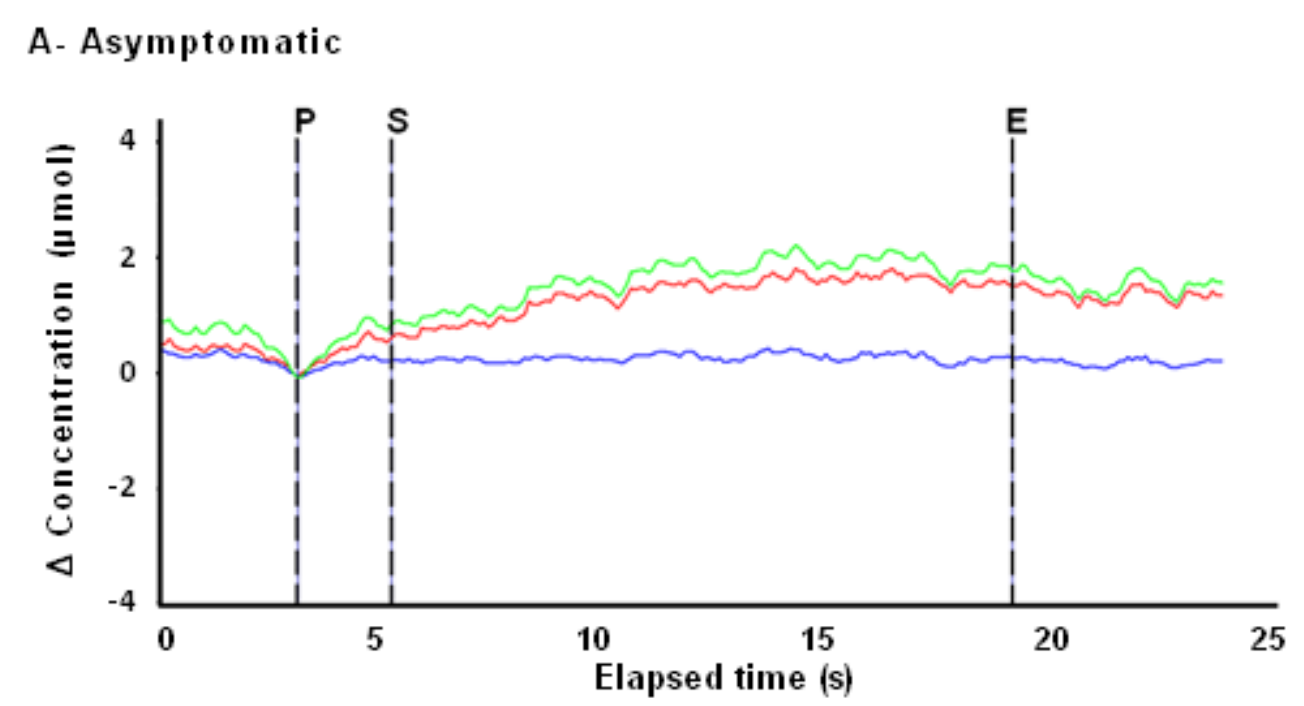

\section{B - NLUTD}

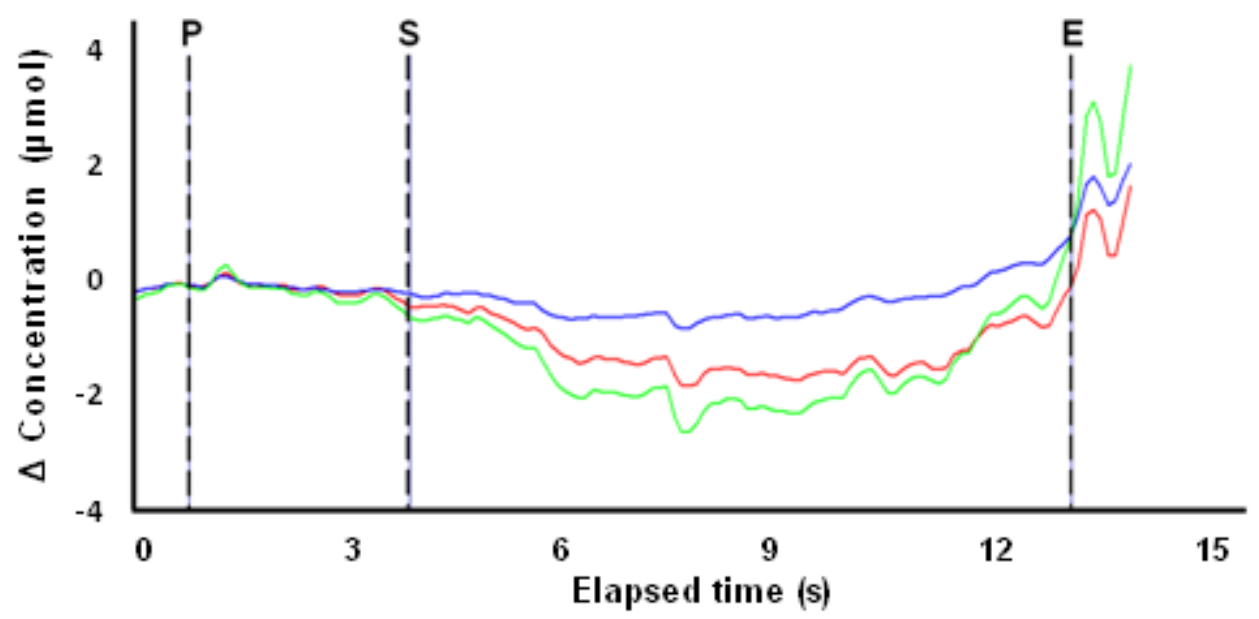

\section{P-Permission to void, S- Urine flow starts, E- Uroflow ends}

Fig. 3. Representative changes in chromophore concentration from permission to void to uroflow end in $A$ : An asymptomatic child and $B$ : A child evaluated for non-neurogenic lower urinary tract dysfunction. The overall trends for $\Delta \mathrm{O}_{2} \mathrm{Hb}$ and $\Delta \mathrm{tHb}$ differ. In $\mathrm{A}$ the pattern and positive trend in $\Delta \mathrm{tHb}$ and $\Delta \mathrm{O}_{2} \mathrm{Hb}$ match chromophore changes seen in asymptomatic adults. In $\mathrm{B}$ There is no rise in total hemoglobin following permission to void and during voiding $\Delta \mathrm{tHb}$ declines and $\mathrm{O}_{2} \mathrm{Hb}$ falls. This negative trend in symptomatic children is compatible with a decrease in detrusor blood volume and the availability of $\mathrm{O}_{2} \mathrm{Hb}$ during voiding. NLUTD: non-neurogenic lower urinary tract dysfunction.

\section{Discussion}

This study indicates that NIRS monitoring of the bladder is feasible using a wireless device. During voiding, asymptomatic adults had patterns of change in chromophore concentration that matched those in asymptomatic children, and were consistent with results obtained with a laser powered instrument. Pilot data from children suggest that those with NLUTD have a different chromophore pattern to asymptomatic children. Physiologically, this appears due to impairment of detrusor hemodynamics and oxygenation, based on comparable NIRS changes seen in voluntary muscle. If further studies confirm these findings, NIRS may contribute to a greater understanding of the physiology underlying their 


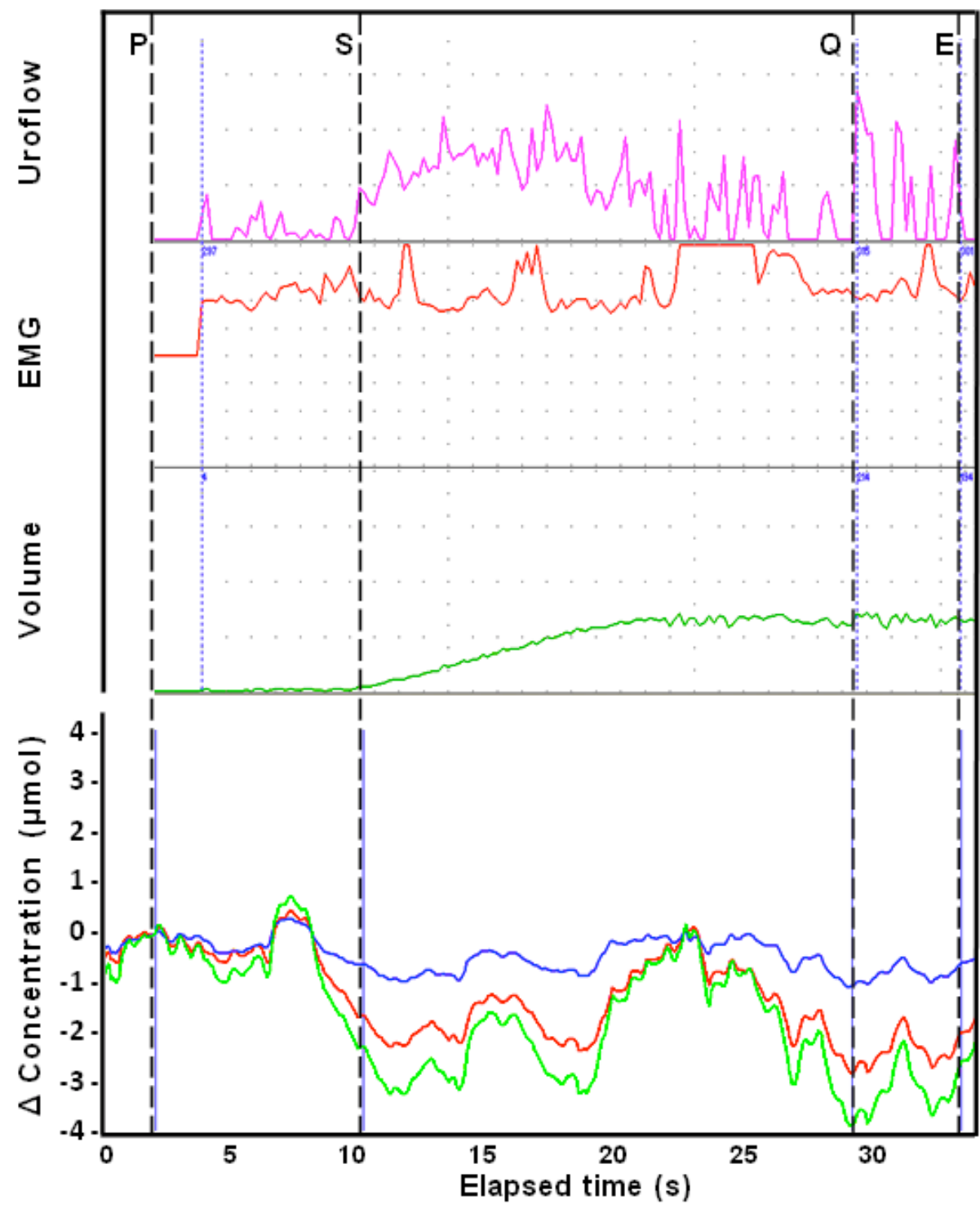

Dysfunctional Voiding

Uroflow Summary:

Max. flow:

$33.7 \mathrm{ml} / \mathrm{s}$

Mean flow:

$8.2 \mathrm{ml} / \mathrm{s}$

Voiding time:

315

Acceleration:

$1 \mathrm{ml} / \mathrm{s}$

Flow time:

$23 \mathrm{~s}$

Voided Volume: $190 \mathrm{ml}$

PVR:

$19 \mathrm{ml}$

P- Permission to void

S- Urine flow starts

Q- Peak urine flow

E- Uroflow ends

Total Hb

Deoxygenated Hb

oxygenated $\mathrm{Hb}$

Fig. 4. Simultaneous data (uroflow, EMG, voided volume, and NIRS pattern of chromophore change in a child with symptoms of non-neurogenic lower urinary tract dysfunction. Following permission to void an initial increase in $\Delta \mathrm{tHb}$ occurs due to an equal increase of $\Delta \mathrm{O}_{2} \mathrm{Hb}$ and $\Delta \mathrm{HHb}$; a sharp decrease in $\Delta \mathrm{tHB}$ is then evident predominantly due to a fall in $\Delta \mathrm{O}_{2} \mathrm{Hb}$ that continues as uroflow starts, periodic increases in $\Delta \mathrm{tHB} / \Delta \mathrm{O}_{2} \mathrm{Hb}$ occur, but the trend is negative during voiding particularly from 23 seconds, while $\Delta \mathrm{HHb}$ remains stable. This chromophore pattern implies a failure of oxygen supply to meet demand following permission to void followed by hemodynamic fluctuation with variations in the availability of $\mathrm{O}_{2} \mathrm{Hb}$ and total blood volume in the detrusor during voiding.

symptoms of urgency, weak flow and retention of urine.

Confidence for NIRS monitoring physiologic change in the detrusor comes from the physics of spectroscopy, chromophore changes only being detected over the bladder during voiding events, and data mirroring observations in other tissue (e.g., during hypoxia or ischemia)., ${ }^{4,16}$ Natural voiding requires detrusor contraction, which in turn requires provision of sufficient oxygenated blood to meet oxygen demand. ${ }^{5}$ And it is becoming evident that where factors exist that adversely affect detrusor muscle function or the blad- der's microcirculation, different patterns of chromophore change are observed during voiding to those seen in asymptomatic subjects. Such patterns include a falling $\Delta \mathrm{O}_{2} \mathrm{Hb}$ and/ or increasing $\Delta \mathrm{HHb}$ (which implies altered oxygen supply/ demand), and compromised $\mathrm{tHb}$ (indicative of impaired hemodynamics). ,, $6-8^{-8}$

In this context, data from the subject with a "shy" bladder provide additional evidence that NIRS-derived chromophore changes reflect physiologic variations occurring in the detrusor microcirculation rather than artifact or movement. 
Between permission to void and uroflow start, the patient was unable to void in spite of sustained urge, most probably associated with spontaneous isovolumetric bladder contraction of low magnitude, and no significant change in bladder size or the organ's position would have occurred as the volume of urine in his bladder did not alter. Yet a sequence of changes in chromophore concentration was observed that clearly shows variations in $\Delta \mathrm{tHb}$ and in $\Delta \mathrm{O}_{2} \mathrm{Hb}$ and $\Delta \mathrm{HHb}$. Based on our current understanding of the microcirculation's role in regulating oxygen supply and demand, ${ }^{17}$ these changes would reflect variations in detrusor hemodynamics and oxygenation. This data sequence and elements of the chromophore change seen in the 6 symptomatic subjects reported (Fig. 3, Fig. 4) correspond to data from voluntary muscle, where variations in blood volume or increased oxygen consumption resulting in oxygen debt are generated experimentally. ${ }^{1-3,18,19}$

We recognize limitations in this report. It only has single observations in 14 asymptomatic subjects (including 4 children) and 6 symptomatic children; the magnitude of chromophore change varies between subjects; and the data analysis compared patterns of change rather than mathematical criteria. However, the patterns of change derived from the two NIRS devices were directly comparable, and the physics principles and algorithms employed in both NIRS devices were the same. ${ }^{13}$ Also, variations in chromophore magnitude are seen in NIRS studies of other tissues, and likely represent differences in light penetration and scattering between trials. ${ }^{2,18}$ Analysis was made of the patterns of change because the duration of voiding naturally differed $s$ between subjects and for each individual void which makes data sets difficult to correlate mathematically.

\section{Conclusion}

NIRS monitoring, when interpreted in the context of change in detrusor oxygenation or hemodynamics, should be of interest to urologists. We observed that subjects with symptomatic prostatic obstruction had little or no increase in $\mathrm{tHb}$ or $\mathrm{O}_{2} \mathrm{Hb}$ throughout their attempts to void. ${ }^{4,7-9}$ This observation parallels NIRS studies of striated muscle where muscle fatigue and symptoms of cramping occur with chromophore changes, suggesting curtailment of oxygen delivery ${ }_{i}^{18}$ and situations in subjects with congestive heart failure where work of breathing increases during exercise associated with respiratory muscle hypo-perfusion. ${ }^{2}$ Therefore, looking at the physiologic basis for NIRS data changes occurring in voiding dysfunction is of greater potential value than reliance on algorithms with diagnostic potential.

There is a need for non-invasive devices and methodology to evaluate voiding dysfunction, particularly in children. As this pilot physiologic data indicate, NIRS can add data of potential value. There is a high level of acceptance of the device by children and wireless devices make ambulant monitoring possible. We suggest NIRS has a role in future applications of urologic research and in diagnostic evaluation.

Competing interests: None declared.

This paper has been peer-reviewed.

\section{References}

1. Wolf $M$, Ferrari $M$, Quaresima V. Progress of near-infrared spectroscopy and topography for brain and muscle clinical applications. J Biomed Opt 2007;12:062104. http://dx.doi.org/10.1117/1.2804899

2. Hamaoka T, McCully KK, Quaresima V, et al. Near-infrared spectroscopy/imaging for monitoring muscle oxygenation and oxidative metabolism in healthy and diseased humans. J Biomed Opt 2007;12:062105. http://dx.doi.org/10.1117/1.2805437

3. Ferrari M, Mottola L, Quaresima V. Principles, techniques and limitations of near infrared spectroscopy. Can J Appl Physiol 2004;29:463-87. http://dx.doi.org/10.1139/h04-031

4. Macnab AJ, Stothers L. Near infrared spectroscopy (NIRS): Validation of a new non-invasive method for evaluation/classification of males with low urinary tract symptoms (LUTS). Can J Urol 2008; 15:4241-340.

5. Macnab AJ, Shadgan B, Stothers L. Dynamic topography of the human bladder during voiding using functional near infrared spectroscopy. J Biomed Opt 2009;14:020507. http://dx.doi.org/10.1117/1.3122886

6. Pannek J, Editorial Comment on: Classification of Male Lower Urinary Tract Symptoms Using Mathematical Modeling and a Regression Tree Algorithm of Noninvasive Near-Ifrared Spectroscopy Parameters. Euro Urol 2010;57:332-3. http://dx.doi.org/10.1016/..eururo.2009.05.005

7. Stothers L, Guevara R, Macnab AJ. Classification of male lower urinary tract symptoms using mathematical modeling and a regression tree algorithm of non-invasive near infrared spectroscopy parameters. Euro Urol 2009; http://dx.doi.org/10.1016/i.eururo.2009.05.005.

8. Te AE, Chung DE, Lee RI, Kaplan SA. Near infrared spectroscopy (NIRS) for application in urology. J Urol 2009;181:601. http://dx.doi.org/10.1016/S0022-5347(09)61689-X

9. Chung DE, Lee RK, Kaplan SA, et al. Concordance of near infrared Spectroscopy with pressure flow studies in men with lower urinary tract symptoms. J Urol 2010;184:2434-9. http://dx.doi.org/10.1016/i. juro.2010.08.027

10. Guevara R, Stothers L, Macnab AJ. Mathematical modeling methodology for generation of a diagnostic algorithm using near-infrared data. Spectroscopy 2011;25:1-11. http://dx.doi.org/10.3233/SPE2010-0486.

11. Farag FF, Martens FM, D'Hauwers KW, et al. Near-Infrared Spectroscopy: A novel, noninvasive, diagnostic method for detrusor overactivity in patients with overactive bladder symptoms - A preliminary and experimental study. Eur Urol 2011; http://dx.doi.org/10.1016/i.eururo.2010.12.032.

12. Marques A, Stothers L, Macnab AJ. The Status of Pelvic Floor Muscle Training for women. Can Urol Assoc J 2010;4:419-24.

13. Van der Sluijs MC, Colier WNJM, Houston RJF, et al. A new and highly sensitive continuous wave near infrared spectrophotometer with multiple detectors. Proc SPIE 1977;3194:63-72. http://dx.doi. org/10.1117/12.301097

14. Shadgan B, Reid WD, Gharakhanlou R, et al. Wireless near-infra-red spectroscopy of skeletal muscle oxygenation and hemodynamics during exercise and ischemia. Spectroscopy 2009;23:233-41.

15. Afshar K, Mirbagheri A, Scott H, et al. Development of a symptom score for dysfunctional elimination syndrome. J Urol 2009;182(4 Suppl):1939-43. hitp://dx.doi.org/10.1016/i.juro.2009.03.009

16. Shadgan B, Afshar K, Stothers L, et al. Near-infrared spectroscopy of the bladder: a new technique for studying lower urinary tract function in health and disease. Proc SPIE 2010;7548, 754804U-1.

17. Ellis CG, Jagger J, Sharpe M. The microcirculation as a functional system. Critical Care 2005;9(Suppl 4):S3-S8. http://dx.doi.org/10.1186/cc3751

18. Boushel R, Langberg $\mathrm{H}, 0$ lesen J, et al. Monitoring tissue oxygen availability with near infrared spectroscopy (NIRS) in health and disease. Scand J Med Sci Sports 2001;11:213-22. http://dx.doi.org/10.1034/ j.1600-0838.2001.110404.x

19. Hampson NB, Piantadosi CA. Near infrared monitoring of human skeletal muscle oxygenation during forearm ischemia. J Appl Physiol 1988;64:2449-57.

Correspondence: Dr. Babak Shadgan, UBC Hospital Bladder Care Centre, Unit IB - Room F329, 2211 Wesbrook Mall, Vancouver, BC V6T 1Z3 ; fax: 604-822-7591; shadgan@alumni.ubc.ca 